\title{
Repetitive Transcranial Magnetic Stimulation (rTMS) for Decreasing Gambling Craving in Patients with Gambling Disorder: A Call for Advanced Clinical Investigations
}

\author{
Chidiebere Emmanuel Okechukwu
}

Sapienza University of Rome, Rome, Italy

\begin{abstract}
Gambling disorder (GD) is an addictive behavioral disorder that alters the frontostriatal neural circuitry and prefrontal control of reward-associated brain areas. An intrusion between prefrontal cortex and the mesolimbic reward pathway has been proposed as the major mechanism behind the pathogenesis of GD. Repetitive transcranial magnetic stimulation (rTMS) is a noninvasive treatment that utilizes magnetic fields to stimulate nerve cells linked to mood and behavioral control; this stimulation is usually applied either on the left or right side of the dorsolateral prefrontal cortex of the brain. rTMS selectively modulates the activities of brain circuits and possess the ability to overturn the alterations in the neurocircuitry of the brain linked to the pathophysiology of GD. rTMS adjusts impulsivity, cognitive/attentional control, cognitive plasticity, and decision-making, which are crucial in decreasing gambling craving and relapse. However, innovative clinical investigations are needed to analyze and establish the impact of rTMS on gambling craving and cessation, using a larger sample size.
\end{abstract}

Keywords: gambling craving; gambling disorder; repetitive transcranial magnetic stimulation; neuroregulation; neurotherapy

Citation: Okechukwu, C. E. (2021). Repetitive transcranial magnetic stimulation (rTMS) for decreasing gambling craving in patients with gambling disorder: A call for advanced clinical investigations. NeuroRegulation, 8(1), 69-71. https://doi.org/10.15540/nr.8.1.69

*Address correspondence to: Chidiebere Emmanuel Okechukwu, Department of Public Health and Infectious Diseases, Sapienza University of Rome, Piazzale Aldo Moro 5, 00185, Rome, Italy. Email: chidiebere.okechukwu@uniroma1.it

Copyright: (๑ 2021. Okechukwu. This is an Open Access article distributed under the terms of the Creative Commons Attribution License (CC-BY).

\section{Edited by:}

Rex L. Cannon, PhD, SPESA Research Institute, Knoxville, Tennessee, USA

\section{Reviewed by:}

Rex L. Cannon, PhD, SPESA Research Institute, Knoxville, Tennessee, USA

Mark S. Jones, DMin, University of Texas at San Antonio, San Antonio, Texas, USA
Gambling disorder (GD) is a complicated behavioral addiction that affects the frontostriatal neural circuitry and prefrontal control of reward-associated brain areas, a discrepancy between prefrontal cortex (PFC) and the mesolimbic reward pathway has been proposed as the major mechanism behind the pathogenesis of GD (Pettorruso et al., 2020). There is an elevated functional connectivity between the regions of the PFC and mesolimbic reward pathway in GD patients (Pettorruso et al., 2020). The distorted connection between prefrontal structures and the mesolimbic reward system in GD is similar to the pathophysiologic mechanism implicated in substances use disorders (Pettorruso et al., 2020). GD is a growing public health issue, and its aftereffects are family issues, substance misuse, suicidal ideation, suicide, and criminal activities such as robbery and drug smuggling to raise cash for gambling (Okechukwu, 2019). Individuals with GD often encounter financial difficulty and abject poverty because of relentless gambling (Okechukwu, 2019). Moreover, mood disorders such as major depressive disorder, generalized anxiety disorder, and personality disorder are associated psychiatric comorbidities of GD (Okechukwu, 2019).

Repetitive transcranial magnetic stimulation (rTMS) is a noninvasive treatment that utilizes magnetic fields to stimulate nerve cells linked to mood and behavioral control, this stimulation is usually applied either on the left or right side of the dorsolateral prefrontal cortex (DLPFC) of the brain (Lefaucheur et al., 2014). High-frequency rTMS of the left DLPFC appears to be effective, and low-frequency rTMS of the right DLPFC has possible effectiveness (Lefaucheur et al., 2014). According to the study 
conducted by Gay et al. (2017), they found that patients with GD had decreased gambling craving following a single session of high frequency rTMS delivered to their left DLPFC after viewing a gambling video, using a MagPro X100 stimulation system.

A key advantage of rTMS is that it selectively modulates the activities of brain circuits and possesses the ability to overturn the alterations in the neurocircuitry of the brain linked to the pathophysiology of GD (Pettorruso et al., 2021). rTMS significantly modulates impulsivity, cognitive/attentional control, decision-making, and cognitive plasticity, which are crucial in decreasing gambling craving and relapse (Zucchella, Mantovani, Federico, Lugobani, \& Tamburin, 2020). rTMS has been shown to adjust dopaminergic and glutamatergic neurotransmission, and both transmissions are altered in GD patients (Pettorruso et al., 2019). rTMS causes a decrease in dopamine transporter availability in striatal regions, leading to modulation in dopaminergic pathways; this implies that rTMS has the potential to modulate brain functioning, and neural circuits associated with GD (Pettorruso et al., 2019).

Neuroimaging findings have shown similar activation defects in regions of the mesolimbic reward system in patients with GD and substance use disorders (Limbrick-Oldfield, Van Holst, \& Clark, 2013). Some patients with GD often abuse cocaine (Cowlishaw, Merkouris, Chapman, \& Radermacher, 2014). However, rTMS is effective in lowering cocaine use and sleep disruption (Gómez Pérez et al., 2020).

Providing $5-\mathrm{Hz}$ rTMS therapy for 2 weeks significantly reduced craving and impulsivity, and improved functional connectivity between left DLPFC with ventromedial prefrontal cortex, and ventromedial prefrontal cortex with right angular gyrus in patients with cocaine use disorder (GarzaVillarreal et al., 2021). Moreover, clinical improvements were observed in patients with comorbid gambling and cocaine use disorder who had undergone a high-frequency left-DLPFC-rTMS stimulation. including diminished cocaine craving and intake and decrease in the craving to gamble. There was also improvement in sleep quality and decrease in anxiety and depression among the patients (Cardullo et al., 2019).

In conclusion, GD is an addictive behavioral disorder, in which dysfunctions in the prefrontal neural activity have been proposed as the underlying pathophysiological mechanism.
Therefore, targeting the PFC for the treatment of GD using rTMS is a promising therapeutic innovation. rTMS seems to decrease gambling craving, enhance mood control, and improve cognitive function in patients with GD. rTMS could emerge as a reliable adjunct therapy in support of cognitivebehavioral therapy for the treatment of GD. However, advanced clinical investigations are needed to scrutinize and establish the impact of rTMS on gambling craving and cessation, using a larger sample size.

\section{Author Disclosure}

There was no financial support received for this study, and there are no conflicts of interest.

\section{References}

Cardullo, S., Gomez Perez, L. J., Marconi, L., Terraneo, A., Gallimberti, L., Bonci, A., \& Madeo, G. (2019). Clinical improvements in comorbid gambling/cocaine use disorder (GD/CUD) patients undergoing repetitive transcranial magnetic stimulation (rTMS). Journal of Clinical Medicine, 8(6), 768. https://doi.org/10.3390/jcm8060768

Cowlishaw, S., Merkouris, S., Chapman, A., \& Radermacher, H. (2014). Pathological and problem gambling in substance use treatment: A systematic review and meta-analysis. Journal of Substance Abuse Treatment, 46(2), 98-105. https://doi.org /10.1016/j.jsat.2013.08.019

Garza-Villarreal, E. A., Alcala-Lozano, R., Fernandez-Lozano, S. Morelos-Santana, E., Dávalos, A., Villicaña, V., ... GonzalezOlvera, J. J. (2021). Clinical and functional connectivity outcomes of $5-\mathrm{Hz}$ repeated transcranial magnetic stimulation as an add-on treatment in cocaine use disorder: A doubleblind randomized controlled trial. Biological Psychiatry: Cognitive Neuroscience and Neuroimaging, S2451. 9022(21)00021-5. Advance online publication. https://doi.org /10.1016/j.bpsc.2021.01.003

Gay, A., Boutet, C., Sigaud, T., Kamgoue, A., Sevos, J., Brunelin, J., \& Massoubre, C. (2017). A single session of repetitive transcranial magnetic stimulation of the prefrontal cortex reduces cue-induced craving in patients with gambling disorder. European Psychiatry, 41(1), 68-74. https://doi.org /10.1016/j.eurpsy.2016.11.001

Gómez Pérez, L. J., Cardullo, S., Cellini, N., Sarlo, M., Monteanni, T., Bonci, A., ... Madeo, G. (2020). Sleep quality improves during treatment with repetitive transcranial magnetic stimulation (rTMS) in patients with cocaine use disorder: A retrospective observational study. BMC Psychiatry, 20(1), 153. https://doi.org/10.1186/s12888-020-02568-2

Lefaucheur, J.-P., André-Obadia, N., Antal, A., Ayache, S. S., Baeken, C., Benninger, D. H., ... Garcia-Larrea, L. (2014). Evidence-based guidelines on the therapeutic use of repetitive transcranial magnetic stimulation (rTMS). Clinical Neurophysiology, 125(11), 2150-2206. https://doi.org /10.1016/j.clinph.2014.05.021

Limbrick-Oldfield, E. H., van Holst, R. J., \& Clark, L. (2013). Fronto-striatal dysregulation in drug addiction and pathological gambling: Consistent inconsistencies? Neurolmage: Clinical, 2, 385-393. https://doi.org/10.1016 /j.nicl.2013.02.005

Okechukwu, C. E. (2019). Role of exercise in the treatment of gambling disorder. Nigerian Journal of Experimental and Clinical Biosciences, 7(1), 50-54. https://doi.org/10.4103 /njecp.njecp_11_19 
Pettorruso, M., Di Giuda, D., Martinotti, G., Cocciolillo, F., De Risio, L., Montemitro, C., .. NST Study Group (2019). Dopaminergic and clinical correlates of high-frequency repetitive transcranial magnetic stimulation in gambling addiction: A SPECT case study. Addictive Behaviors, 93, 246-249. https://doi.org/10.1016/j.addbeh.2019.02.013

Pettorruso, M., Martinotti, G., Montemitro, C., De Risio, L., Spagnolo, P. A., Gallimberti, L., ... Brainswitch Study Group (2020). Multiple sessions of high-frequency repetitive transcranial magnetic stimulation as a potential treatment for gambling addiction: A 3-month, feasibility study. European Addiction Research, 26(1), 52-56. https://doi.org/10.1159 1000504169

Pettorruso, M., Miuli, A., Di Natale, C., Montemitro, C., Zoratto, F., De Risio, L., ... di Giannantonio, M. (2021). Non-invasive brain stimulation targets and approaches to modulate gambling-related decisions: A systematic review. Addictive Behaviors, 112 , /j.addbeh.2020.106657

Zucchella, C., Mantovani, E., Federico, A., Lugoboni, F., \& Tamburin, S. (2020). Non-invasive brain stimulation for gambling disorder: A systematic review. Frontiers in Neuroscience, 14, $729 . \quad$ https://doi.org/10.3389 /fnins.2020.00729

Received: January 25, 2021

Accepted: February 13, 2021

Published: March 29, 2021 